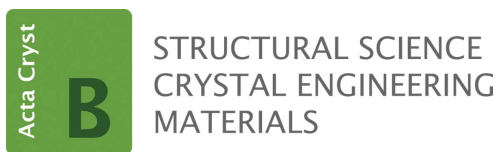

ISSN 2052-5206

Keywords: electron crystallography; electron diffraction; data processing

\section{Introduction to the special issue on electron crystallography}

\author{
Joke Hadermann $^{\mathrm{a} *}$ and Lukáš Palatinus ${ }^{\mathrm{b} *}$ \\ a Department of Physics, University of Antwerp, Groenenborgerlaan 171, Antwerp, 2020, Belgium, and ${ }^{\mathbf{b}}$ Institute of \\ Physics of the AS CR, v.v.i, Cukrovarnicka 10, 16200 Prague 6, Czech Republic. *Correspondence e-mail: \\ joke.hadermann@uantwerpen.be,palat@fzu.cz
}

Structure analysis of micro- and nanocrystalline materials has witnessed immense progress in the last decade thanks to the development of electron diffraction techniques. The automation of data collection, development of new data collection modes and improvements in the data treatment have allowed unprecedented progress in most aspects of crystallography dealing with very small crystals. Probably the most notable change of paradigm is observable in the structure determination of unknown phases by electron diffraction. Three-dimensional diffraction techniques now allow almost routine solution and refinement of structures from single crystals as small as a few tens of nanometres, providing access to hitherto unsolvable crystal structures or to previously unattainable level of structural detail. Scanning diffraction techniques allow phase and orientation mapping with nanometre resolution and even three-dimensional reconstruction of phase and orientation distributions.

This special issue features a collection of original contributions covering a broad range of aspects of electron crystallography. An interested reader will find papers describing the foundations and methodological basis of structure solution by electron diffraction (Eggeman, 2019; Kolb et al., 2019; Gemmi \& Lanza, 2019), theoretical and methodological advances in data processing (Rauch \& Véron, 2019; Palatinus et al., 2019; Latychevskaia \& Abrahams, 2019), discussion of applications of electron diffraction outside the realm of perfectly periodic crystals (Gorelik et al., 2019; Mugnaioli \& Gorelik, 2019) as well as specific case studies showing the application of the methods to hot topics in current crystallography (Wang et al., 2019; Hadermann \& Abakumov, 2019).

The collection of contributions in this special issue showcases the diversity of applications of current electron diffraction techniques, demonstrates the state of the development of the technique and also features work that further advances the electron diffraction methods. We believe that this special issue can serve as a starting point for anybody interested in electron crystallography and we are convinced that the contributions in this issue will become reference points for future research in this exciting field.

\section{References}

Eggeman, A. S. (2019). Acta Cryst. B75, 475-484.

Gemmi, M. \& Lanza, A. E. (2019). Acta Cryst. B75, 495-504.

Gorelik, T. E., Neder, R., Terban, M. W., Lee, Z., Mu, X., Jung, C., Jacob, T. \& Kaiser, U. (2019). Acta Cryst. B75, 532-549.

Hadermann, J. \& Abakumov, A. M. (2019). Acta Cryst. B75, 485-494.

Kolb, U., Krysiak, Y. \& Plana-Ruiz, S. (2019). Acta Cryst. B75, 463-474.

Latychevskaia, T. \& Abrahams, J. P. (2019). Acta Cryst. B75, 523-531.

Mugnaioli, E. \& Gorelik, T. E. (2019). Acta Cryst. B75, 550-563.

Palatinus, L., Brázda, P., Jelinek, M., Hrdá, J., Steciuk, G. \& Klementová, M. (2019). Acta Cryst. B75, 512-522.

Rauch, E. \& Véron, M. (2019). Acta Cryst. B75, 505-511.

Wang, W., Zhou, X., Yang, Z., Qi, Y. \& Ye, H. (2019). Acta Cryst. B75, 564-569. 